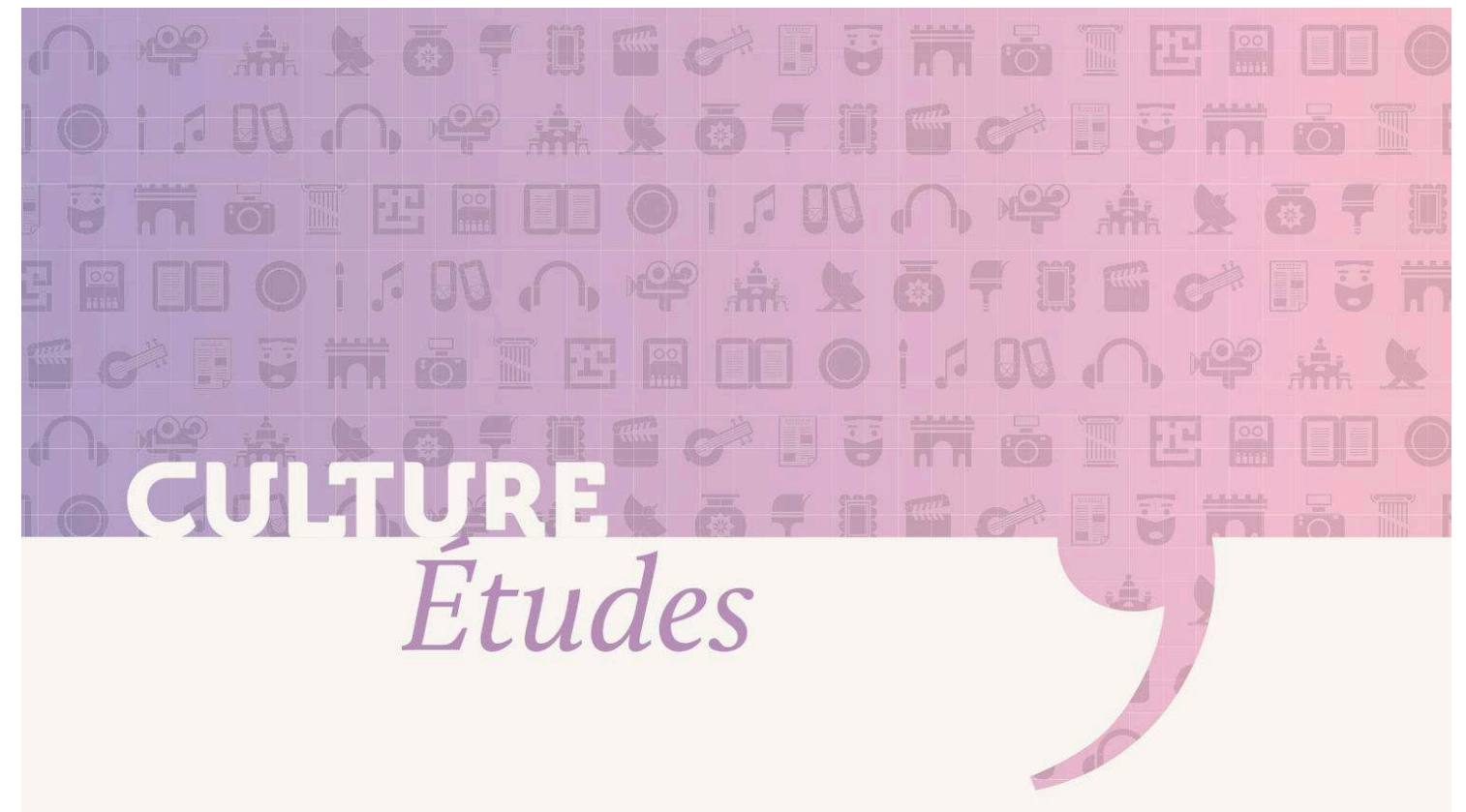

Collectionneurs d'art contemporain : des acteurs méconnus de la vie artistique

Nathalie Moureau Dominique Sagot-Duvauroux Marion Vidal
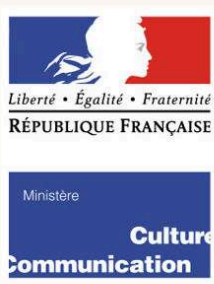

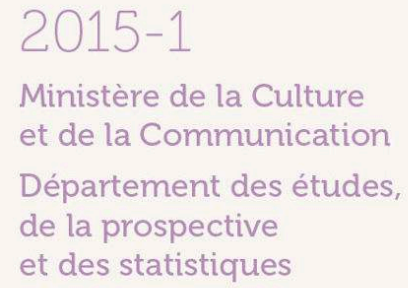

et des statistiques 


\section{Collectionneurs d'art contemporain : des acteurs méconnus de la vie artistique}

Contemporary Art Collectors: Unsung Influential Figures on the Art Scene

Nathalie Moureau, Dominique Sagot-Duvauroux et Marion Vidal

Éditeur : Département des études, de la prospective et des statistiques

Lieu d'édition : Paris

Année d'édition : 2015

Date de mise en ligne : 8 juillet 2015

Collection : Culture études

ISBN électronique : 9782111399143

\section{Sbooks}

http://books.openedition.org

\section{Édition imprimée}

Date de publication : 1 avril 2015

Nombre de pages : 22

\section{Référence électronique}

MOUREAU, Nathalie ; SAGOT-DUVAUROUX, Dominique ; et VIDAL, Marion. Collectionneurs d'art contemporain : des acteurs méconnus de la vie artistique. Nouvelle édition [en ligne]. Paris : Département des études, de la prospective et des statistiques, 2015 (généré le 25 avril 2021). Disponible sur Internet : <http://books.openedition.org/deps/907>. ISBN : 9782111399143. 


\section{CUIDSF Etudes}

\section{Collectionneurs d'art contemporain : des acteurs méconnus de la vie artistique}

Nathalie Moureau Dominique Sagot-Duvauroux Marion Vidal

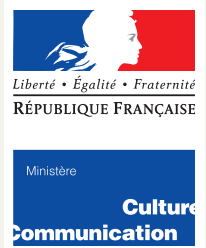

2015-1

Ministère de la Culture et de la Communication Département des études, de la prospective et des statistiques 


\title{
Collectionneurs d'art contemporain : des acteurs méconnus de la vie artistique
}

\author{
Nathalie Moureau* \\ Dominique Sagot-Duvauroux ${ }^{* *}$ \\ et Marion Vidal ${ }^{* * *}$
}

Qu'est-ce qu'un collectionneur? Si le nom a fait officiellement son entrée dans le dictionnaire en $1789^{1}$, sa définition recouvre des pratiques différentes : certaines approches mettent en avant la valeur subjective attachée à l'objet, tandis que d'autres soulignent l'importance du processus d'accumulation et de sélection. De nombreux traits semblent pouvoir définir communément les collectionneurs, quel que soit l'objet de la collection, coquillage, timbre, papillon ou encore œuvre d'art. Toutefois, les collectionneurs qui soutiennent l'art «en train de se faire » se distinguent par des pratiques plus variées que celles usuellement relevées chez leurs homologues. En effet, en plus d'être acquéreur, le collectionneur d'art contemporain est susceptible d'agir sur la vie artistique, notamment grâce au soutien matériel apporté aux artistes et en contribuant à la construction de la valeur artistique. Cette particularité est d'autant plus notable que l'engagement du collectionneur n'est pas l'apanage d'une seule élite fortunée, comme le montre la typologie des profils de collectionneurs établie ici. Il apparaît en effet que les pratiques de collectionneurs se déploient le long d'un continuum allant du collectionneur acquéreur, mû par le besoin de posséder une œuvre, au collectionneur acteur, pour lequel l'acquisition n'est que l'expression de sa volonté de contribuer à la vitalité de la scène artistique.

\footnotetext{
* Maître de conférences à l'université Paul-Valéry de Montpellier et membre du LAMETA (UMR 5474). ** Professeur à l'université d'Angers et membre du GRANEM (UMR 49).

*** Docteur en sciences économiques de l'université de Montpellier et chercheur associée au LAMETA (UMR 5474), université Paul-Valéry, Montpellier.

1. «Collectionneur, euse. s. (on prononce les deux I). Celui, celle qui fait des collections. Collection. s. f. Réunion de plusieurs objets qui ont ensemble quelque rapport. II a une belle collection de tableaux, de livres, d'antiques, de médailles, de plantes, de coquilles, etc. » Dictionnaire de l'Académie française, 1789 (7édition).
} 


\section{Les collectionneurs de la scène contemporaine, profils et parcours}

\section{Diplômés, seniors et franciliens}

La population des collectionneurs d'art actuel telle qu'elle ressort de l'enquête réalisée (voir Élément de méthodologie, p. 18) est majoritairement masculine (73\%) et se distingue par son niveau de diplôme, plus élevé que l'ensemble de la population française puisque les trois quarts des collectionneurs sont titulaires d'un diplôme de niveau bac +4 (graphique 1 ). Un peu plus d'un quart des collectionneurs (27\%) sont titulaires d'un diplôme en histoire de l'art.

Près des deux tiers des collectionneurs (64\%) sont âgés de plus de 50 ans et près de la moitié résident en Île-de-France (47\%) (graphique 2). Ces caractéristiques rejoignent celles des publics de la culture. II existe en effet une forte corrélation entre la détention d'un diplôme de l'enseignement supérieur et la fréquentation de musées et de galeries, ceux-ci étant par ailleurs plus prisés des personnes résidant dans les grandes villes. En outre, l'âge moyen élevé des collectionneurs s'explique, en partie, par la capacité financière liée au cycle de vie : acheter des œuvres suppose de disposer d'un certain budget, ce qui exclut, de fait, une grande partie de la population jeune.

\section{Graphique 1 - Répartition des collectionneurs selon le niveau de qualification}

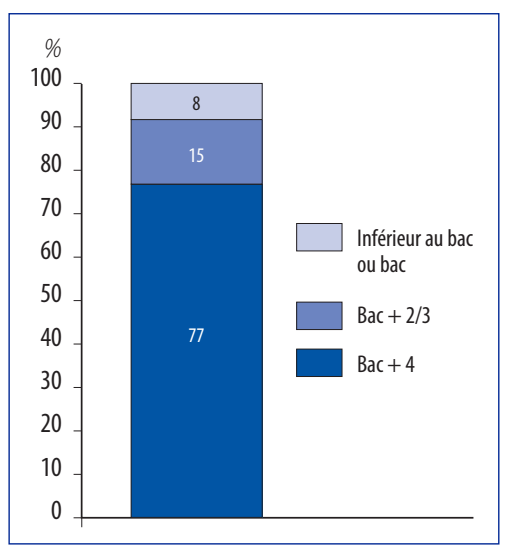

Source: Ministère de la Culture et de la Communication, 2015

\section{Graphique 2 - Répartition des collectionneurs selon l'âge}

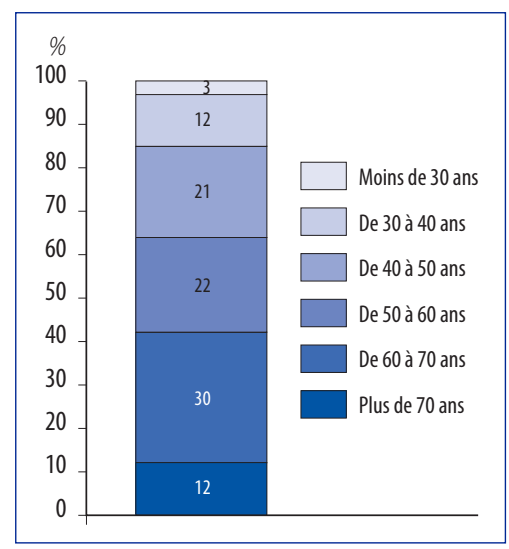

Source : Ministère de la Culture et de la Communication, 2015

\section{Des parcours de collectionneurs diversifiés}

Chaque collectionneur se souvient de la première œuvre acquise, mais l'âge auquel a été faite cette première acquisition varie : $43 \%$ d'entre eux avaient alors entre 20 et 30 ans et un tiers des personnes ont commencé leur collection plus tôt encore. Pour près de la moitié, la première œuvre acquise est une peinture, un tiers ont porté leur choix sur une estampe, une gravure ou une lithographie et ils ne sont qu'un sur dix à avoir acquis un dessin (graphique 3). L'environnement familial est un facteur déterminant puisqu'un tiers des collectionneurs ont connu dans leur environnement familial des personnes qui collectionnaient, et $8 \%$ ont eu des parents exerçant une profession liée au monde de l'art, professions qui ne représentent pourtant que $2 \%$ de la population active. 


\section{Graphique 4 - Part du temps de loisir dédié à la collection}

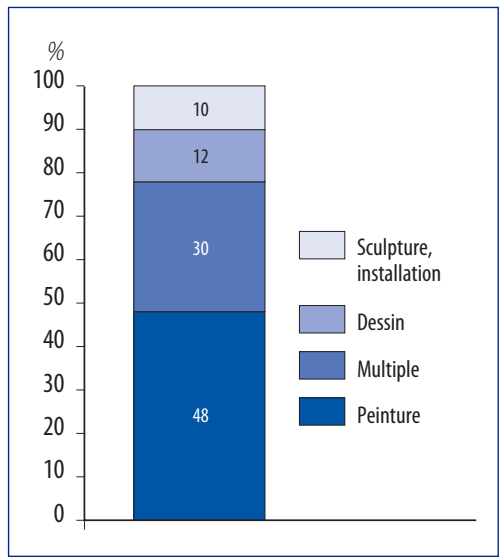

Source: Ministère de la Culture et de la Communication, 2015

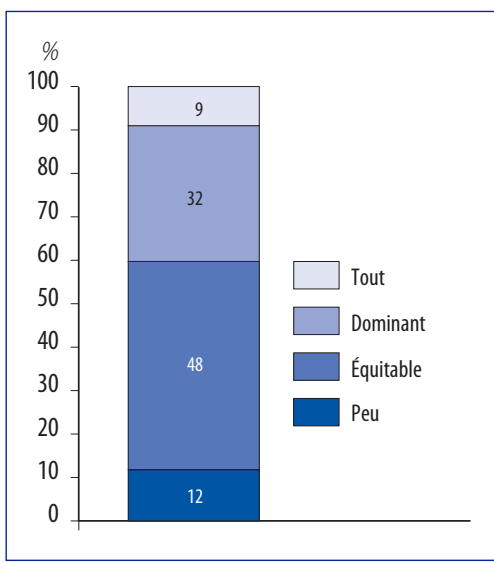

Source : Ministère de la Culture et de la Communication, 2015

Constituer une collection nécessite un investissement non seulement financier mais également temporel et humain : il faut recueillir de l'information, fréquenter les expositions, participer à la vie artistique en suivant l'actualité des expositions, etc. Ainsi, un tiers des collectionneurs consacrent la majorité de leur temps libre à leur collection, et $9 \%$ y affectent même tout leur temps libre (graphique 4). Seul un faible pourcentage des personnes interrogées $(7 \%)$ occupe une partie de ce temps à la réalisation et à l'actualisation d'un site internet en lien avec la collection.

\section{Des collections de taille et de nature variées}

Des plus petites collections, constituées de moins de 50 pièces, qui représentent un tiers de l'ensemble de l'échantillon, aux plus grandes, qui réunissent plus de 200 pièces (une collection sur cinq), l'éventail de la taille des collections est très large (graphique 5). Elles sont pour la plupart exposées au domicile du collectionneur. Un peu plus d'un quart des collectionneurs exposent également des œuvres sur leur lieu de travail $(27 \%)$, enfin une faible proportion ( $8 \%$ ) ont même un lieu d'exposition dédié. Si un quart ( $27 \%$ ) des collectionneurs disposent d'un lieu de réserve spécifique, le domicile reste le principal lieu de stockage des œuvres non exposées.

La population cible de l'enquête étant les personnes collectionnant des œuvres réalisées par des artistes qui leur

Graphique 5 - Taille des collections selon le nombre de pièces possédées

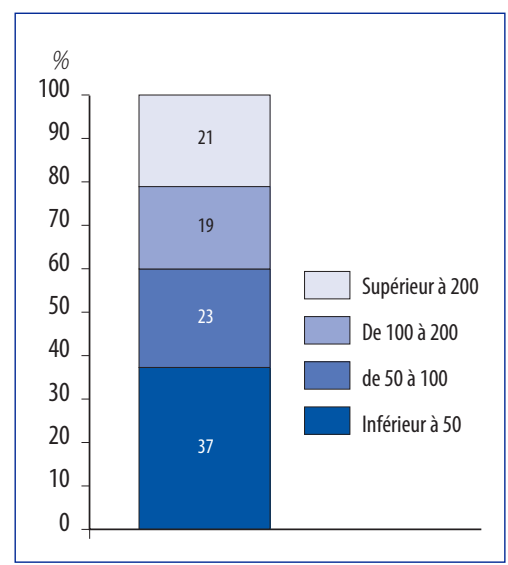

Source : Ministère de la Culture et de la Communication, 2015 
sont contemporains, une très large proportion des œuvres qu'ils détiennent a été réalisée après 1945 (68\%). Dans l'ensemble, les collections ne sont pas spécialisées (66\%) et, pour les trois quarts d'entre elles, sont constituées en majorité d'œuvres d'artistes français ou vivant en France. Une collection sur dix compte exclusivement des œuvres d'artistes français.

S'agissant des supports, la peinture est présente dans presque toutes les collections ( $90 \%$ des cas), la sculpture, la photographie et le dessin dans près des trois quarts, les livres d'artistes dans près de la moitié des collections, tandis que vidéos et installations sont plus rarement représentées (graphique 6).

\section{Graphique 6 - Nature des œuvres possédées}

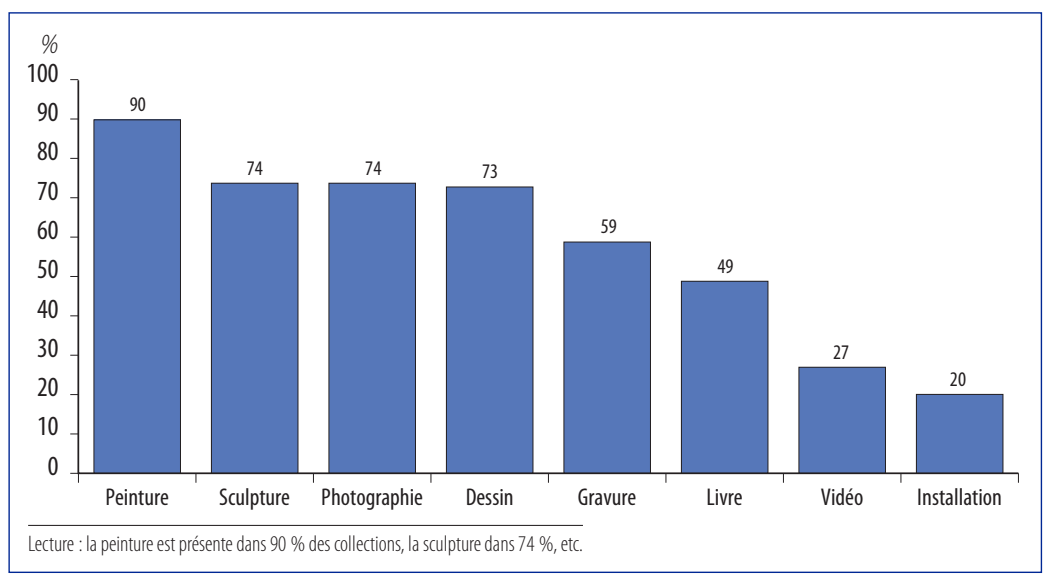

Source : Ministère de la Culture et de la Communication, 2015

\section{Des engagements financiers variables}

Le budget moyen annuel d'acquisition varie considérablement (graphique 7): $30 \%$ des collectionneurs consacrent à l'achat d'œuvres moins de 5000 euros par an, tandis qu'à l'autre extrémité du spectre, $16 \%$ dépensent plus de 50000 euros. Rapporté à leurs revenus annuels, les trois quarts des collectionneurs affectent chaque année l'équivalent d'au moins un mois de leurs revenus à leurs acquisitions, et un quart y affecte même plus de l'équivalent de deux mois de revenus (graphique 8). Le montant maximal dépensé pour l'achat d'une œuvre confirme la diversité des profils des collectionneurs: un quart d'entre eux n'ont jamais dépensé plus de 5000 euros pour une œuvre, tandis qu'un sur dix a déjà acquis une œuvre pour plus de 100000 euros (graphique 9).

\section{Graphique 7 - Budget annuel moyen consacré à l'acquisition d'œuvre(s)}

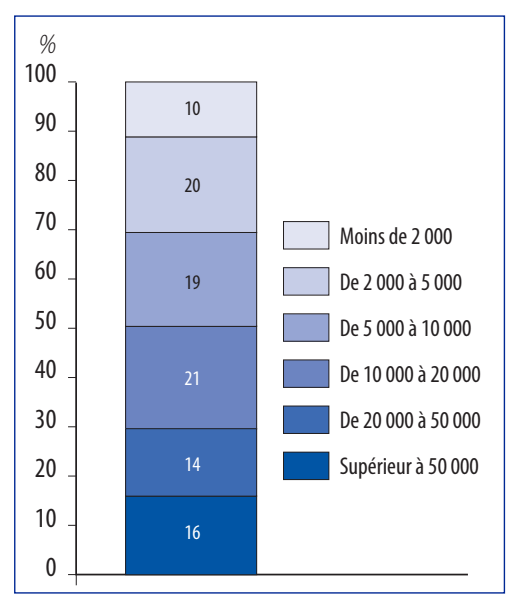

Source: Ministère de la Culture et de la Communication, 2015 


\section{Graphique 8 - Part des revenus annuels moyens dédiés à l'acquisition d'œuvre(s)}

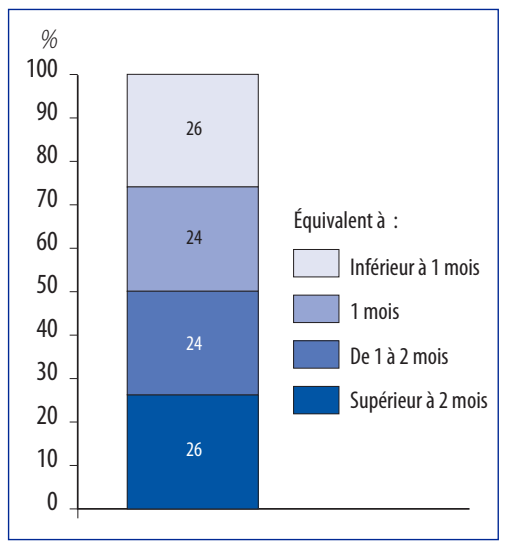

Source : Ministère de la Culture et de la Communication, 2015
Graphique 9 - Somme maximale consacrée à l'acquisition d'une œuvre

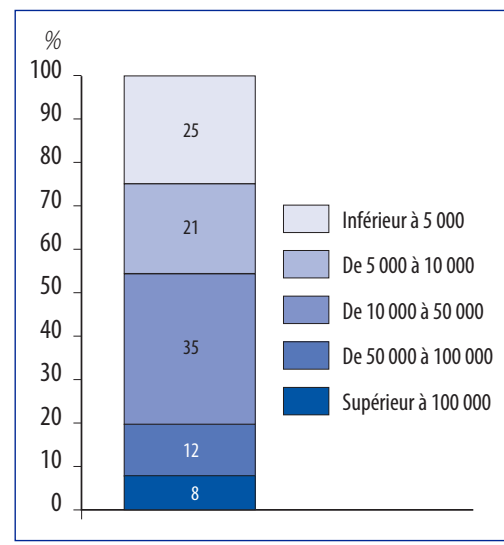

Source : Ministère de la Culture et de la Communication, 2015

\section{Collectionneurs : quels engagements dans la vie artistique?}

Le collectionneur d'art contemporain n'est pas un simple acquéreur. Il est présent sur les deux faces du marché et opère non seulement du côté de la demande, mais également du côté de l'offre à travers son engagement dans la vie artistique. Ainsi, plus des trois quarts des collectionneurs interviennent sous différentes formes pour soutenir les acteurs de l'écosystème de l'art. L'investissement du collectionneur peut concerner la production (commande, financement de catalogue, etc.), la diffusion (prêts pour exposition, présentation à d'autres collectionneurs, etc.) ou l'assistance (soutien financier, matériel, etc.). Les actions du collectionneur peuvent être orientées vers plusieurs acteurs : I'artiste, la galerie ou encore l'institution.

\section{Engagement auprès des artistes : assistance, soutien à la production, soutien à la diffusion}

L'engagement des collectionneurs auprès des artistes prend différentes formes : assistance, aide à la production ou à la diffusion. Plus de la moitié des collectionneurs (54\%) déclarent s'être impliqués dans au moins une de ces formes de soutien à la création artistique (tableau 1).

\section{Assistance}

Un tiers des collectionneurs apportent un soutien matériel ou financier aux artistes. Lorsqu'il est matériel, le soutien se traduit principalement par la mise à disposition de locaux (atelier ou hébergement) et par la fourniture de matériaux. Lorsqu'il est financier, il renvoie aux dons, prêts et avances que le collectionneur effectue pour dépanner l'artiste en difficulté. Cette aide relève parfois d'un don/contre-don ou s'assimile à un don/achat quand l'artiste donne, en retour du soutien reçu, une œuvre à son mécène. L'achat régulier d'œuvres à l'artiste est une autre pratique dont l'objectif prioritaire consiste à assurer à l'artiste des revenus récurrents, les œuvres acquises ne correspondant pas toujours à ce qu'aurait acheté le collectionneur s'il avait été dégagé de cette contrainte de soutien. 
Quelques cas plus originaux de soutien financier ont pu être relevés lors des entretiens conduits auprès des collectionneurs, comme la collecte de fonds auprès d'un réseau d'amis. En contrepartie de cette aide, qui a permis à l'artiste de produire des œuvres en vue d'une exposition, les contributeurs ont reçu un dessin. Ces différentes formes d'assistance ne sont pas exclusives et peuvent se cumuler, l'accompagnement pouvant être d'ordre matériel, financier mais aussi moral.

\section{Soutien à la production}

Les commandes constituent une autre forme d'engagement du collectionneur et $44 \%$ d'entre eux ont déclaré en avoir passé à des artistes. Certes, certaines de ces commandes relèvent de situations traditionnelles d'achat, comme c'est le cas quand il s'agit de posséder une œuvre personnalisée, par exemple un portrait. Mais elles peuvent aussi traduire un désir d'accompagnement, comme lorsque l'œuvre commandée fait ensuite l'objet d'une exposition spécifique. La volonté de soutien prime alors en général sur l'aspect patrimonial, la démarche d'achat n'étant pas le but ultime. $32 \%$ des collectionneurs ont déclaré avoir participé au financement de la production d'œuvre à des fins d'exposition dans des galeries ou en institutions.

\section{Soutien à la diffusion}

Au-delà de l'aide matérielle ou financière (par le prêt ou l'aide à la production), certaines actions conduites par les collectionneurs contribuent à asseoir la notoriété des artistes et/ou à faire émerger de nouveaux talents, comme dans le cas d'une collaboration avec un commissaire d'exposition. Ainsi, $35 \%$ des collectionneurs ont déclaré

Tableau 1 - Comportements des collectionneurs selon différentes formes d'engagement auprès des

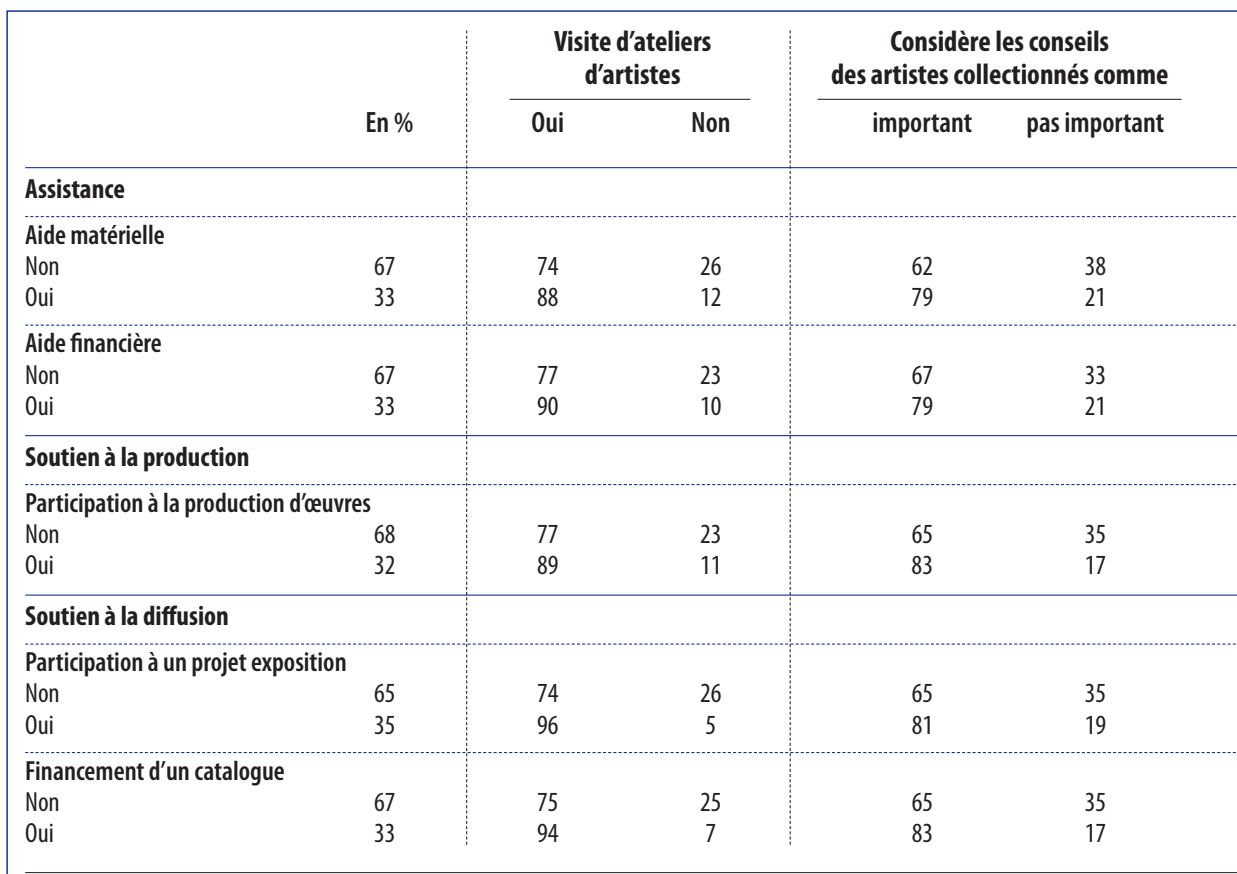

Note de lecture : $33 \%$ des collectionneurs apportent une aide matérielle aux artistes ; parmi ceux-ci, $88 \%$ visitent des ateliers d'artistes, $79 \%$ considèrent les conseils des artistes collectionnés comme 
avoir déjà collaboré à un projet d'exposition avec un commissaire d'exposition ou un critique et $33 \%$ avoir publié ou participé au financement de catalogues d'artistes présents dans leur collection. La multiplicité des modalités selon lesquelles les collectionneurs sont susceptibles de s'engager dans un projet d'exposition est apparue au cours des entretiens. Ce peut être la création, la location ou le financement d'un lieu, le prêt de son propre appartement ou encore la collaboration avec les institutions. Dans certains cas, le collectionneur lui-même peut endosser le rôle de commissaire d'exposition.

Les modalités d'engagement des collectionneurs peuvent aussi être d'ordre symbolique, en faisant notamment bénéficier les artistes de leur capital social. En leur ouvrant leur réseau, ils élargissent leur marché potentiel (mise en contact avec d'autres collectionneurs) et peuvent contribuer à une plus grande légitimité artistique (introduction auprès de galeristes, d'institutionnels). Dans certains cas, l'artiste peut également bénéficier du réseau professionnel du collectionneur, notamment lorsque ce dernier a constitué une collection d'entreprise destinée à faire découvrir les œuvres à ses salariés et aux partenaires de l'entreprise. L'aide à la diffusion peut également se traduire sous des formes plus singulières lorsqu'un collectionneur endosse le rôle de manager d'artistes en les accompagnant dans leurs démarches administratives et financières, dans la recherche de lieux de vente ou d'exposition, voire en les assistant dans leur comptabilité. Cet accompagnement peut également prendre la forme d'une activité de communication : rédaction d'articles de presse consacrés aux artistes de leur collection ou financement de campagnes de publicité.

artistes

\begin{tabular}{|c|c|c|c|c|c|c|c|}
\hline \multicolumn{2}{|c|}{$\begin{array}{l}\text { Taille de la collection selon } \\
\text { le nombre d'œuvres possédées }\end{array}$} & \multicolumn{3}{|c|}{$\begin{array}{c}\text { Part du temps libre } \\
\text { consacrée à la collection }\end{array}$} & \multicolumn{3}{|c|}{ Achat direct à l'artiste } \\
\hline$<100$ & $>100$ & dominant & équitable & faible & régulier & parfois & jamais \\
\hline 78 & 22 & 32 & 52 & 16 & 23 & 49 & 28 \\
\hline 45 & 55 & 48 & 43 & 9 & 43 & 49 & 8 \\
\hline 69 & 31 & 36 & 50 & 14 & 27 & 51 & 23 \\
\hline 43 & 57 & 48 & 44 & 8 & 47 & 46 & 7 \\
\hline 72 & 28 & 35 & 51 & 14 & 30 & 49 & 21 \\
\hline 36 & 64 & 51 & 42 & 8 & 41 & 50 & 10 \\
\hline 76 & 29 & 32 & 54 & 15 & 26 & 59 & 22 \\
\hline 41 & 59 & 54 & 37 & 9 & 46 & 46 & 9 \\
\hline 73 & 27 & 36 & 51 & 13 & 28 & 50 & 21 \\
\hline 35 & 65 & 49 & 40 & 11 & 44 & 47 & 9 \\
\hline
\end{tabular}




\section{Collectionneurs et artistes : une nécessaire rencontre?}

Près des trois quarts des collectionneurs rencontrent les artistes dont ils achètent les œuvres; pour un collectionneur sur cinq, cette démarche est systématique. Différents motifs sont à l'origine de cette volonté de connaître l'artiste: pouvoir échanger, comprendre les œuvres et la démarche artistique. La relation permet aussi de conforter ses choix. Pour d'autres collectionneurs au contraire, seule l'œuvre compte, et celle-ci prime sur l'artiste.

Pour autant, la personnalité de l'artiste, les rapports financiers ou encore le fait que l'œuvre dépasse l'artiste peuvent atténuer ce besoin de rencontre. Le temps peut également contribuer à ternir la relation, lorsque le collectionneur se sent piégé et obligé d'acheter une œuvre du fait de la proximité qu'il entretient avec l'artiste. Certains collectionneurs s'interrogent ainsi sur la nature de l'engagement de l'artiste dans une telle relation, et craignent que l'intérêt qui leur est témoigné relève de considérations financières et matérielles plus que de liens singuliers.

Les collectionneurs engagés auprès des artistes ont une propension plus élevée que les autres à visiter des ateliers d'artistes, à accorder de l'attention aux conseils que leur prodiguent les artistes et à s'adresser directement à eux pour leurs achats d'œuvres (tableau 1). Par exemple, $46 \%$ des collectionneurs qui ont participé à un projet d'exposition déclarent acheter directement à l'artiste de façon régulière contre seulement $26 \%$ pour ceux qui n'ont pas participé à de tels projets. De même, ils sont $96 \%$ à visiter les ateliers d'artistes et $81 \%$ à considérer les conseils d'artistes comme importants, contre respectivement $74 \%$ et $65 \%$ pour les autres.

Ces collectionneurs engagés auprès des artistes consacrent un temps très important à leur collection : près de la moitié de ceux qui déclarent apporter une aide matérielle à l'artiste affectent aussi la majeure partie de leur temps libre à leur collection, une proportion qui ne concerne qu'un tiers (32\%) de ceux qui n'apportent pas d'aide financière aux artistes.

La corrélation financière ou matérielle entre l'investissement financier sous la forme de financement de catalogue et la taille de la collection s'observe également. Les deux tiers des collectionneurs (65\%) ayant participé au financement d'un catalogue possèdent plus de 100 œuvres, contre un quart ( $27 \%)$ pour ceux qui n'ont jamais participé à ce type de financement.

Pour autant, s'ils sont relativement plus nombreux à considérer l'avis des artistes comme important quand ils accordent une aide financière à l'artiste (61\% contre $47 \%$ ), ceux qui participent à la production d'œuvres et au financement de catalogues semblent plus indépendants dans leurs choix et affichent des motivations distinctes. Une plus faible proportion accorde ainsi de l'importance aux conseils des commissaires d'exposition et des galeristes.

Que l'on considère l'aide à la production, la participation à un projet d'exposition ou encore la participation au financement de catalogue, environ 6 collectionneurs sur 10 ayant effectué l'une de ces actions déclarent accorder de l'importance à la démarche, contre 4 collectionneurs n'ayant pas développé ce type de soutien.

\section{Engagement auprès des institutions muséales}

Au travers des relations qu'ils entretiennent avec les institutions, les collectionneurs sont susceptibles d'apporter indirectement un soutien plus ou moins conséquent aux artistes.

\section{Sociétés d'amis de musée, prêt d'œuvres, participation à une commission d'achat d'œuvres...}

Une des formes les plus fréquentes d'engagement est l'adhésion à une société d'amis de musées : $60 \%$ des collectionneurs en sont membres (tableau 2). Si cette 
adhésion leur permet d'accéder à divers services et privilèges (visites d'ateliers, invitation à des vernissages, etc.), les cotisations versées contribuent en retour à soutenir financièrement l'institution concernée.

Plus de la moitié des collectionneurs prêtent des œuvres à des musées. $27 \%$ ont effectué ces prêts exclusivement en France, $29 \%$ en France et à l'étranger. Les prêts exclusivement internationaux ne concernent que $2 \%$ des collectionneurs.

On observe enfin des formes d'engagement plus spécifiques qui requièrent souvent une collaboration plus étroite avec l'institution : participation au conseil d'administration d'un musée ou à une commission d'achat (14\%) ou encore dons ( $8 \%$ ) ou dépôts d'œuvres ( $8 \%$ ) au sein d'un musée. La réglementation qui encadre certaines de ces pratiques contribue sans doute pour partie au caractère plus confidentiel de cette activité qui suppose, en outre, une étroite collaboration entre l'institution et le collectionneur (encadré « Encadrement juridique des dons et des dépôts d'œuvres dans un musée »). Au-delà, le fait d'avoir eu sa première œuvre jeune (avant 20 ans) a une influence positive sur les dons ou dépôts ( $46 \%$ des collectionneurs impliqués), les prêts en France et à l'étranger (42\%) alors que cela n'impacte pas le fait de participer administrativement à la vie d'un musée (société d'amis ou conseil d'administration).

\section{Encadrement juridique des dons et dépôts d'œuvres dans un musée}

Outre les dispositions destinées à favoriser l'enrichissement des collections publiques après qu'est intervenu le décès des collectionneurs (procédure de la dation et mesures fiscales accompagnant les legs), diverses mesures encadrent les relations du vivant du collectionneur (Code du patrimoine), notamment pour ce qui concerne les dons et les dépôts effectués auprès des musées de France.

Pour qu'une œuvre issue d'une collection privée puisse être déposée dans un musée, l'avis de la Commission scientifique des musées nationaux est préalablement requis. Un contrat est alors établi précisant les conditions du dépôt auquel est annexé l'état de l'œuvre. La durée du dépôt ne peut être inférieure à cinq ans. Il est possible de la prolonger d'un an par avenant. Le contrat peut également prévoir les conditions selon lesquelles le propriétaire peut retirer l'œuvre déposée pour une durée limitée après accord du dépositaire. Bien évidemment, ces dépôts sont effectués à titre gracieux.

Le don d'œuvres à un musée, manuel ou sous acte notarié, ouvre à des avantages fiscaux. Un montant équivalent à $66 \%$ du montant du don (la valeur de l'œuvre étant évaluée par un expert) peut être déduit du montant de l'impôt sur le revenu, à hauteur de $20 \%$ du revenu imposable. Dans le cas où le don excéderait $20 \%$ du revenu imposable du collectionneur, un report du solde peut être effectué sur les cinq années suivantes. Par ailleurs, selon l'article 1131 du Code général des impôts, les dons manuels d'œuvres au profit de l'État sont exonérés de droits de mutation. Notons que le donataire peut inclure diverses clauses à son don et spécifier notamment les conditions de présentation et de conservation de l'œuvre. Dans le cas particulier d'une donation avec réserve d'usufruit, le donateur conserve la jouissance de l'œuvre sur une période prédéfinie contractuellement.

\section{Des comportements différents selon la forme d'engagement du collectionneur}

Les collectionneurs engagés auprès des institutions consacrent, comparativement aux autres, plus de leur temps libre à leur passion. Corrélée à leur engagement, la taille de leur collection est plus élevée, notamment lorsqu'ils effectuent des prêts en France et à l'étranger (63\% des prêteurs détiennent ainsi plus de 100 pièces). Leur 
budget est également conséquent, la part des collectionneurs consacrant un budget supérieur à 50000 euros est de l'ordre de $30 \%$ pour les collectionneurs ayant effectué des dons, des dépôts ou des prêts en France et à l'étranger, et tombe à $6 \%$ pour ceux n'ayant pas ce type d'engagement.

Quelle que soit la forme que prend leur relation avec les institutions, les collectionneurs engagés ont tendance à être plus nombreux à visiter régulièrement les foires étrangères que les non-engagés. Cet écart peut même aller du simple au double pour les collectionneurs membres d'une société d'amis de musée.

Les conseils extérieurs d'un commissaire d'exposition sont jugés importants pour les collectionneurs membres d'un comité d'administration ou d'une commission d'achat et pour les collectionneurs membres de sociétés d'amis de musée (respectivement $73 \%$ et $61 \%$ ), de même que pour ceux qui effectuent des dons ou des dépôts (63\%). Ces derniers se distinguent des autres par la détention de collections exclusivement constituées d'œuvres produites après 1945 (57 \%), par le fait de ne pas posséder d'autres collections que des œuvres d'art (56\%) et de posséder majoritairement des œuvres d'artistes français ou vivant en France (65\%).

\section{Collectionneurs et institutions, entre attirance et frustration}

La plupart des collectionneurs citent un musée ou une exposition visitée au cours de leur jeunesse comme déclic de leur passion. Beaucoup évoquent aussi l'importance de leurs rencontres avec des conservateurs dans leur formation artistique. Cependant, leurs rapports avec les institutions sont complexes. D'un côté, ils considèrent comme une consécration et comme une reconnaissance de leur engagement le fait que leurs choix artistiques soient confirmés par un musée, qu'ils soient sollicités pour faire partie d'un conseil d'administration ou d'une commission d'achat ou encore pour prêter une œuvre. De l'autre, ils regrettent parfois le manque d'égards des institutions vis-àvis de leur concours, notamment dans le cas de prêt d'œuvres. De ce point de vue, les

Tableau 2 - Comportements des collectionneurs selon la forme d'engagement auprès d'institutions

\begin{tabular}{|c|c|c|c|c|}
\hline & \multirow[b]{2}{*}{ En $\%$} & \multicolumn{3}{|c|}{ Visite foires étrangères } \\
\hline & & Régulièrement & Rarement & Jamais \\
\hline \multicolumn{5}{|c|}{ Membre d'une société d'amis de musée } \\
\hline Non & 30 & 39 & 17 & 44 \\
\hline Oui & 60 & 75 & 11 & 14 \\
\hline \multicolumn{5}{|c|}{$\begin{array}{l}\text { Membre du conseil d'administration } \\
\text { ou de commission d'achat d'un musée ou d'une institution } \\
\text { dédiée à l'art contemporain }\end{array}$} \\
\hline Non & 86 & 56 & 15 & 29 \\
\hline Oui & 14 & 87 & 7 & 7 \\
\hline \multicolumn{5}{|c|}{ Prêt d'œuvres à des institutions pour une exposition } \\
\hline France & 27 & 89 & 5 & 6 \\
\hline France et étranger & 31 & 96 & 3 & 1 \\
\hline aucun & 42 & 79 & 11 & 14 \\
\hline \multicolumn{5}{|c|}{ Dépôt ou don d'œuvres à un musée } \\
\hline Non & 92 & 58 & 15 & 29 \\
\hline Oui & 8 & 76 & 8 & 16 \\
\hline
\end{tabular}

Note de lecture : $60 \%$ des collectionneurs sont membres d'une société d'amis de musée ; parmi ceux-ci, 75 \% visitent régulièrement des foires étrangères, contre 39 \% des collectionneurs 
institutions étrangères sont plutôt mieux jugées, même si neuf collectionneurs sur dix considèrent que ces prêts ont été effectués globalement dans de bonnes conditions en France.

Au-delà, plusieurs collectionneurs regrettent d'être souvent réduits à une fonction de fournisseurs de services ou de pourvoyeurs de fonds et de ne pas être considérés comme des partenaires à part entière, porteurs d'une expertise concurrente ou complémentaire de celle des membres de l'institution. Au collectionneur serait reconnue la fonction de mécène sans la légitimité du conservateur.

\section{Engagement auprès des galeries}

La plupart des collectionneurs suivent le travail d'un nombre restreint de galeries ( $39 \%$ suivent moins de cinq galeries ou lieux d'exposition et $32 \%$, entre cinq et dix), soit parce qu'ils ont établi une relation privilégiée avec quelques galeristes, soit parce qu'ils n'ont pas le temps d'en suivre un plus grand nombre. À la faveur de ces liens de proximité, le collectionneur est parfois conduit à apporter un soutien d'ordre financier à une galerie en difficulté. Ce soutien peut alors prendre deux formes : la première s'apparente à une sorte d'épargne, le collectionneur versant une rente mensuelle au galeriste en contrepartie d'une ou plusieurs pièces; la seconde, plus fréquente, est celle d'achat ponctuel d'œuvres.

L'engagement du collectionneur peut également passer par une participation à l'activité de la galerie; soit de façon ponctuelle par le financement d'un catalogue par exemple, soit de façon plus suivie et étroite comme dans le cas d'un tandem galeriste/collectionneur, le premier proposant les artistes et les idées, le second les moyens financiers nécessaires à l'activité. La collaboration peut aussi être d'ordre intellectuel, le collectionneur proposant des idées ou participant à la vie de la galerie.

muséales

\section{Taille de la collection}

$<100>100$
Temps libre consacré à la collection

Dominant Équitable Faible

\section{Budget moyen consacré à la collection}

$<10000 \quad$ Entre $10>50000$

et 50000

\begin{tabular}{|c|c|c|c|c|c|c|c|}
\hline 73 & 27 & 31 & 47 & 22 & 67 & 25 & 8 \\
\hline 53 & 47 & 46 & 48 & 6 & 38 & 39 & 20 \\
\hline 64 & 36 & 37 & 49 & 14 & 65 & 30 & 16 \\
\hline 41 & 59 & 52 & 43 & 5 & 20 & 63 & 16 \\
\hline 57 & 43 & 42 & 42 & 15 & 42 & 42 & 14 \\
\hline 37 & 63 & 57 & 36 & 7 & 22 & 47 & 31 \\
\hline 80 & 19 & 25 & 60 & 15 & 74 & 20 & 6 \\
\hline & & 36 & 50 & 14 & 56 & 3 & 12 \\
\hline & & 52 & 41 & 7 & 28 & 42 & 31 \\
\hline
\end{tabular}


Collectionneurs et galeristes : "Je t'aime moi non plus »

Les galeristes ont a priori les faveurs de la grande majorité des collectionneurs. Les galeries constituent leur principale source d'approvisionnement, seuls $6 \%$ de collectionneurs déclarent ne jamais y acheter d'œuvres (graphique 10).

Pour nombre de collectionneurs, les galeries ont été le lieu de formation de leur œil. Les conseils des galeristes sont ainsi considérés comme très importants ou importants pour $74 \%$ des collectionneurs (graphique 11 ).

Certains collectionneurs ont déclaré au cours des entretiens qu'ils pouvaient parfois même aller contre leur propre sensibilité première pour suivre les recommandations d'un galeriste en lequel ils avaient confiance. D'autres soulignent comment des relations de fidélité et d'amitié se sont nouées au cours du temps avec quelques galeristes.

La dette que les collectionneurs reconnaissent avoir vis-à-vis de certains galeristes n'exclut pas néanmoins un regard parfois très critique sur la nature financière de leurs liens. Plus largement, la profession est présentée comme non ouverte et peu affable.

\section{Graphique 10 - Fréquentation des lieux d'achat}

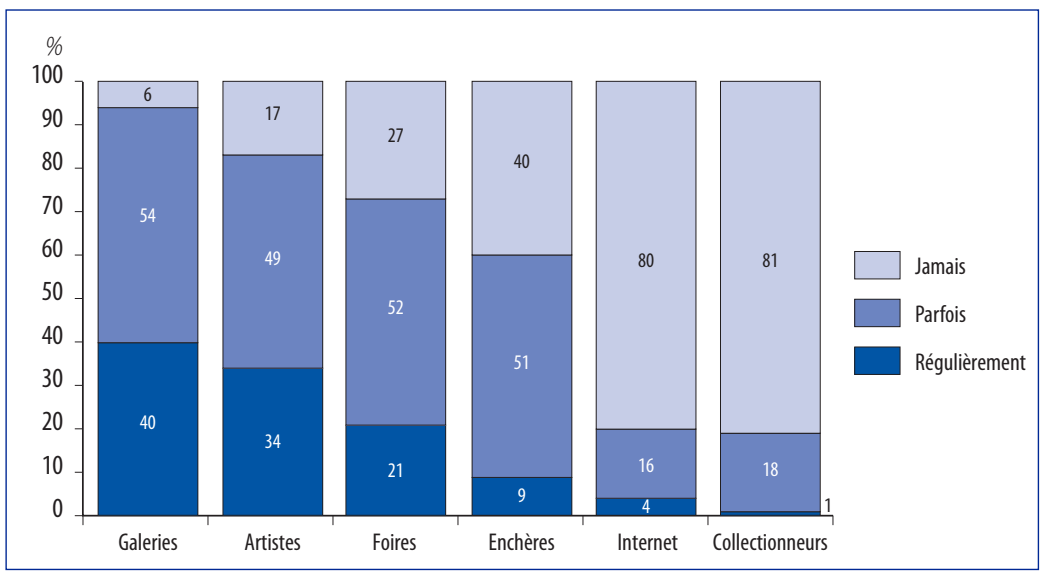

Source: Ministère de la Culture et de la Communication, 2015

\section{Graphique 11 - Conseils considérés comme importants lors de l'achat}

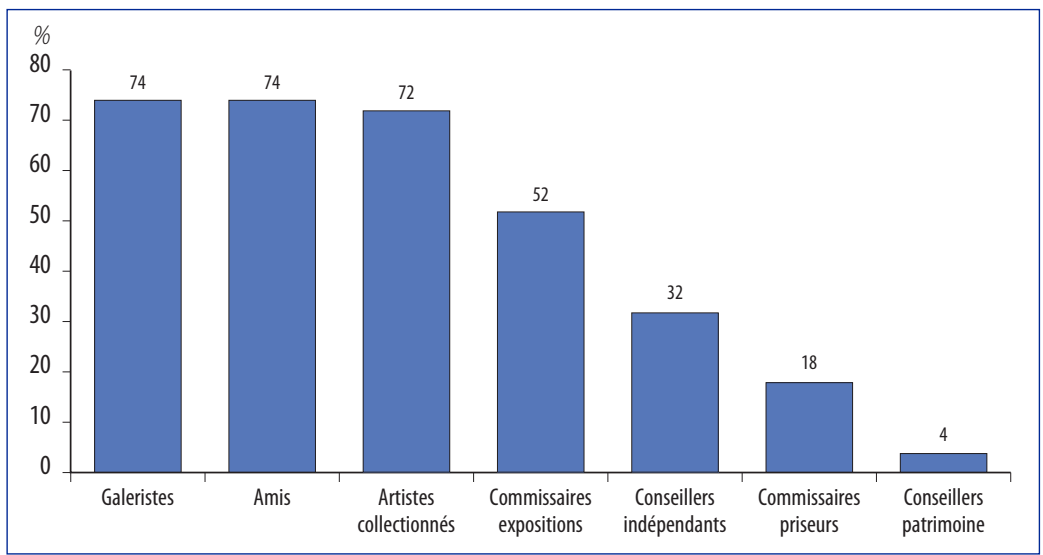

Source : Ministère de la Culture et de la Communication, 2015 


\section{Des formes d'engagement qui se cumulent}

L'engagement des collectionneurs dans le soutien à la vie artistique est généralement pluriel. Les sept formes d'engagement les plus souvent énoncées sont : prêt d'œuvre pour une exposition en institution ; soutien financier ou matériel à l'artiste ; participation au conseil d'administration ou à une commission d'achat d'une institution dédiée à l'art contemporain ; conduite d'un projet d'exposition en collaboration avec un commissaire d'exposition ou un critique ; publication ou participation au financement de catalogues d'artistes; don d'œuvres à un musée ; dépôt d'œuvres dans un musée. Quatre collectionneurs sur cinq sont engagés dans au moins une forme de ces activités. Parmi eux, nombreux sont ceux qui sont engagés dans plusieurs activités : près de $40 \%$ des collectionneurs cumulent ainsi au moins trois formes d'engagement (graphique 12).

\section{Graphique 12 - Nombre d'activités liées à un engagement dans la vie artistique}

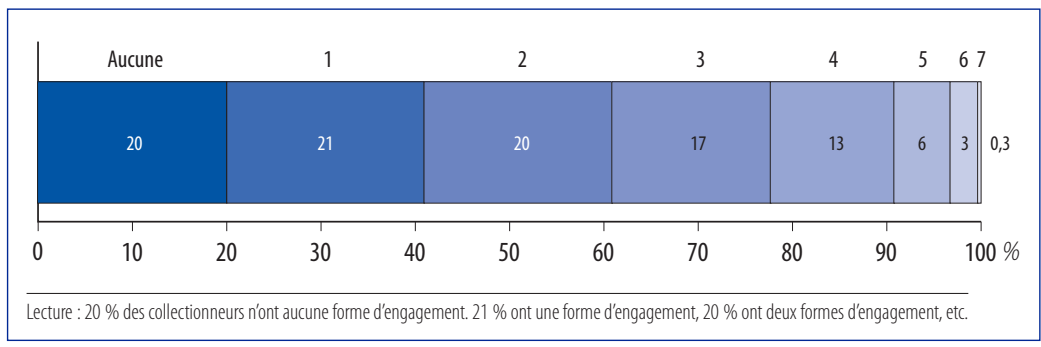

Source: Ministère de la Culture et de la Communication, 2015

Les liens entretenus avec l'institution sont corrélés à l'intensité de l'engagement. Prêts et soutien à l'artiste constituent les premières formes d'engagement (graphique 13). Lorsque le nombre d'actions augmente, les actions se diversifient et s'orientent davantage vers les institutions avec le financement de catalogues, la participation à l'organisation d'expositions, voire l'appartenance à un conseil d'administration ou au comité d'achat d'une institution. Les pratiques de dons et de dépôts restent l'apanage des collectionneurs fortement engagés dans le soutien à la vie artistique, ces formes de soutien ne concernant que les collectionneurs ayant déclaré cinq formes d'engagement ou plus. Sans doute, l'encadrement réglementaire du don et du dépôt soumis à l'acceptation de comités d'experts explique pour partie la moindre diffusion de ces pratiques.

\section{Graphique 13 - Nature des actions} conduites quand l'individu a un seul engagement

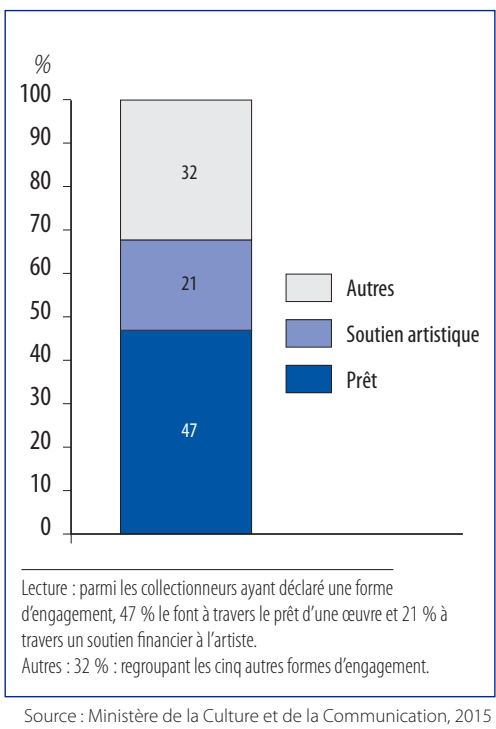




\section{Des formes d'engagement aux motivations variées}

Quatre principaux mobiles sont à l'origine de l'investissement des collectionneurs dans la vie artistique. Si l'altruisme peut être à la source de leur engagement, des mobiles individuels (recherche de plaisir esthétique et culturel), sociaux (désir de se distinguer ou de s'intégrer dans un milieu social convoité) ou encore financiers (s'enrichir, constituer un patrimoine) guident aussi leurs actions. Ces motivations ne sont pas exclusives les unes des autres et leur hiérarchie et l'intensité avec lesquelles elles se manifestent se retrouvent dans des proportions variables selon les collectionneurs.

\section{Engagement altruiste}

L'engagement des collectionneurs dans la vie artistique peut, en premier lieu, répondre à une motivation altruiste. La satisfaction éprouvée par les collectionneurs résulte alors non pas d'une amélioration de leur situation personnelle par suite d'une consommation ou d'une production, mais plutôt du bien-être éprouvé par d'autres individus. Certains collectionneurs déclarent ainsi avoir une responsabilité sociale et soutiennent des artistes, des galeries, non pas pour en retirer une utilité personnelle, mais pour les aider à développer leur activité et/ou à vivre de façon moins précaire. Les soutiens surviennent souvent dans des situations critiques qui menacent la poursuite de l'activité artistique de l'acteur concerné.

L'altruisme peut, par exemple, se concrétiser par le paiement d'un prix supérieur à celui proposé par l'artiste. Les jeunes artistes, en effet, connaissent mal le marché et peuvent proposer des prix soit trop élevés par rapport à leur notoriété, soit trop bas par rapport à ce qui leur est nécessaire pour vivre et pour amortir les coûts. Certains collectionneurs vont alors volontairement proposer à des jeunes artistes un prix d'acquisition plus élevé que celui initialement demandé. Le geste altruiste peut naître d'une proximité esthétique, familiale ou amicale.

\section{Enjeu esthétique et culturel}

L'engagement du collectionneur peut être motivé par la satisfaction personnelle qu'il en retire. Tandis que la confrontation à des œuvres lui permet d'éprouver directement un plaisir artistique et esthétique, son engagement dans la vie artistique lui ouvre la voie vers des satisfactions que l'on peut qualifier de « dérivées », puisqu'il lui permet d'une part d'affiner son goût et d'autre part d'avoir la satisfaction de contribuer à l'art «en train de se faire».

Sociologues et économistes ont souligné le rôle de l'expérience et de l'apprentissage dans la formation du goût. L'engagement peut, pour partie, résulter de la volonté du collectionneur de former son goût. L'accueil d'artistes à son domicile ou dans un atelier dédié lui ouvre une meilleure compréhension de la démarche artistique. Dans le même esprit, côtoyer des professionnels (critiques, commissaires, conservateurs), écouter leur opinion et l'analyse qu'ils font des œuvres lui permettent d'affiner son propre jugement. Plus largement, sa participation aux instances de gouvernance d'une institution ou encore l'organisation d'expositions le renseignent sur les processus de production et de valorisation de la création contemporaine et sur les règles tacites de fonctionnement du monde de l'art. Le collectionneur peut, par ailleurs, retirer une satisfaction du soutien qu'il apporte à la création et de sa participation au processus créatif.

Au-delà, le collectionneur peut s'engager dans la vie artistique parce qu'il considère celle-ci comme une sorte de bien public. Dans ce cas singulier, ce n'est pas le plaisir retiré de la possession de l'œuvre qui prime mais plutôt le fait que l'achat permette de soutenir la création. Par son intervention, il participe à la vitalité de la scène artistique contemporaine et à sa diversité, quelquefois en réaction aux choix jugés trop normés des institutions. Et c'est l'existence même de cette vitalité qui lui procure une satisfaction. 
Finalement, par son engagement dans la vie artistique, le collectionneur se forme (learning by doing), retire un plaisir spécifique à participer au processus créatif et contribue à la diversité de la création.

\section{Enjeu social et distinctif}

Une troisième motivation possible de l'engagement des collectionneurs dans la vie artistique est liée au caractère social de ces activités: la satisfaction retirée de l'engagement est alors plutôt issue des rencontres, du positionnement social et des effets de distinction qu'elles procurent. L'engagement est pour partie lié à la satisfaction retirée à côtoyer un milieu considéré comme valorisant, intéressant, stimulant. Les associations de collectionneurs ou d'amis des musées jouent un rôle important pour favoriser les échanges entre collectionneurs eux-mêmes ou entre collectionneurs et institutions.

L'engagement peut aussi être plus directement lié à une recherche de distinction qui s'obtient de deux façons. La première consiste à avoir des consommations différenciées du reste de la population. Ainsi, il n'est pas rare que le plaisir esthétique retiré de l'appréciation de l'œuvre soit rehaussé par le sentiment de fierté que ressent le collectionneur à faire partie d'une minorité éclairée qui a accès à l'œuvre, qui a su reconnaître avant les autres l'artiste important, se distinguant du reste de la population. Cette recherche de distinction constitue par ailleurs un puissant moteur pour la vitalité de la scène artistique : comme le plaisir s'émousse au fur et à mesure que les œuvres de l'artiste sont diffusées sur le marché, le collectionneur est naturellement conduit à se tourner vers d'autres artistes moins connus et à les promouvoir.

Plus prosaïquement, la distinction peut être obtenue par l'affichage ostentatoire de la collection ou des moyens qui lui sont affectés.

Plus généralement, certains collectionneurs, notamment en région, retirent une réelle satisfaction à apparaître comme acteurs de la vie artistique locale et d'être reconnus comme tels.

\section{Enjeu économique}

Enfin, il serait naif de penser que l'engagement des collectionneurs dans la vie artistique soit totalement déconnecté d'enjeux économiques et financiers. D'abord, cet engagement, notamment auprès des institutions, permet de disposer d'informations privilégiées sur les artistes prometteurs, d'autant plus précieuses que la majorité des collectionneurs, y compris les plus fortunés, se déclarent incapables de suivre l'escalade des prix propre au fonctionnement actuel du marché de l'art.

Ensuite, la position qu'ils occupent sur le marché grâce à leur engagement peut leur permettre de produire eux-mêmes des signaux susceptibles d'affecter la cote des artistes et notamment ceux présents dans leur collection. Ne dit-on pas sous forme de plaisanterie que lorsque François Pinault s'arrête devant une œuvre dans une exposition, la cote de l'artiste concerné en est immédiatement affectée. Certes, peu de collectionneurs bénéficient de cette situation enviable de faire partie des instances de légitimation de l'art, mais le simple fait d'appartenir à une commission d'achat d'une institution accorde un pouvoir d'influence. Qu'un collectionneur utilise sa position institutionnelle pour défendre les artistes qu'il apprécie et qu'il achète est cependant tout à fait légitime s'il est en mesure de convaincre les autres membres de la commission de la pertinence de ses choix. De même, le prêt d'une œuvre dans une exposition à forte notoriété ne peut que valoriser a posteriori la valeur de cette œuvre. 


\section{Vers une typologie des collectionneurs engagés : du quasi-professionnel à l'indépendant}

En plus de s'organiser sur un continuum allant d'actions simples vers des actions de nature plus institutionnelle, l'engagement plus ou moins conséquent des collectionneurs dans le soutien à la vie artistique apparaît lié à certaines variables. La taille de la collection est à cet égard symptomatique : les personnes non engagées dans les activités de production possèdent des collections de moins de 50 œuvres en général, alors que l'engagement dans la production est corrélé à la taille de la collection et s'élargit en direction des institutions au fur et à mesure que la collection grandit. Une analyse des correspondances multiples a permis de faire émerger quatre grands types de collectionneurs caractérisés selon des formes d'engagement distinctes.

\section{Les collectionneurs quasi professionnels ${ }^{2}$}

Un premier groupe rassemble des collectionneurs qui ont un engagement conséquent, notamment dans les activités de production légitimées. Ils ont déjà été impliqués dans la conduite de projets avec des institutions, ont effectué des prêts et dépôts auprès de musées, financé des catalogues et effectué des aides à la production. En parallèle, ils prêtent leurs œuvres tant en France qu'à l'étranger.

Ils agissent quasiment en professionnels, déclarent consacrer tout leur temps libre à leur collection, et ont généralement débuté leur collection avant l'âge de 20 ans. Ils se fient à leur propre jugement et accordent peu de poids au fait que l'œuvre ait été préalablement exposée en galerie ou dans un lieu apprécié. En corollaire, ils ne déclarent pas suivre le travail de galeries en particulier. En outre, le motif de décoration n'est pas jugé comme étant important. Les lieux d'achats fréquentés sont pluriels. Les achats sont régulièrement effectués auprès d'artistes, aux enchères et assez fréquemment à d'autres collectionneurs.

La taille des collections et le pouvoir d'achat associés à ce profil sont conséquents : le prix maximum pour acquérir une œuvre peut être supérieur à 100000 euros et la collection comporte parfois plus de 200 pièces.

\section{Les collectionneurs investis}

Les collectionneurs de cette catégorie sont également investis dans des activités de production mais de façon différente par rapport au groupe des collectionneurs quasi professionnels. Ils sont ainsi sollicités pour participer au conseil d'administration de différentes institutions ou pour prêter des œuvres de leur collection en France et à l'étranger. L'acquisition de leur première œuvre s'est effectuée au début de leur vie d'adulte, entre 20 et 30 ans. Ils affectent la majeure partie de leur temps de loisir à leur collection, sont très actifs dans la recherche d'informations, lisent Art press, fréquentent la foire de Bâle et suivent régulièrement l'activité de cinq à dix galeries, parfois même plus. Ils valorisent la démarche de l'artiste et sont attentifs au fait que l'œuvre qui les attire ait été exposée dans une galerie ou dans un lieu qu'ils apprécient lorsqu'ils ont eu à effectuer un choix. L'avis des critiques compte également pour eux.

Ces collectionneurs s'inscrivent dans les réseaux usuels du marché. S'ils déclarent effectuer régulièrement leurs acquisitions en galerie, ils fréquentent aussi les foires et peuvent parfois acheter aux enchères. En revanche, ils n'ont jamais acheté d'œuvre en ligne.

2. La caractérisation des collectionneurs qui ressort de cette typologie doit être interprétée comme relativement aux autres catégories. Ainsi, lorsque l'on écrit : ils prêtent leurs œuvres tant en France qu'à l'étranger, cela doit être compris par: plus fréquemment que les autres collectionneurs. 
Leurs collections comptent entre 100 et 200 œuvres et le prix d'acquisition le plus élevé qu'ils ont déboursé varie de 10000 à 50000 euros.

\section{Les collectionneurs pondérés}

L'implication dans l'activité de production des collectionneurs pondérés est moindre que celle des quasi-professionnels ou des investis, mais n'est pas absente pour autant. S'ils ne sont pas investis dans la production de catalogue d'exposition et ne sont pas présents dans les conseils d'administration, il leur arrive d'effectuer des prêts auprès d'institutions françaises. Ces collectionneurs, qui ont commencé à collectionner en entrant dans l'âge adulte, entre 20 et 30 ans, déclarent partager équitablement leur temps libre entre leur activité de collection et d'autres loisirs.

À l'image des investis, ils repèrent différents signaux dans leur environnement pour conforter leur jugement sur l'œuvre et suivent régulièrement le travail de quelques galeries, en général moins de cinq. Le fait qu'une œuvre ait été exposée dans un lieu ou une galerie qu'ils apprécient est pour eux un critère de choix important. Plus encore, le fait qu'elle soit apparue aux enchères peut jouer aussi dans certains cas. À la différence des collectionneurs investis, la décoration est très souvent le motif d'achat.

Les lieux d'acquisition des œuvres sont assez usuels : parfois en galeries, rarement directement auprès d'artistes et jamais auprès d'autres collectionneurs.

Leurs collections sont en moyenne de taille un peu plus réduite que celles des deux catégories de collectionneurs précédentes et comptent moins de 50 œuvres qu'ils ont acquises pour un montant maximal compris entre 10000 et 50000 euros.

\section{Les collectionneurs indépendants}

Ces collectionneurs, qui ont commencé leur collection en majorité après la trentaine, ne sont pas impliqués dans des activités de production et n'effectuent pas de prêts de leurs œuvres. Ils n'affectent qu'une part réduite de leur temps libre à leur activité de collection, sont peu investis dans la recherche d'information, ne suivent pas de galeries en particulier mais peuvent en revanche fréquenter des foires localisées en région. Ils sont assez autonomes dans leur jugement, accordent peu ou pas de poids au fait que l'œuvre ait été préalablement exposée dans des lieux ou des galeries qu'ils apprécient et déclarent ne pas être influencés par les avis des critiques. Leurs acquisitions sont motivées par le choix d'une œuvre plutôt que par une démarche d'artiste.

Les réseaux mobilisés pour l'achat d'œuvres sont innovants : si les galeries ne sont pas forcément dédaignées, le web constitue un lieu d'achat assez prisé, tandis que ces collectionneurs indépendants ne fréquentent pas les ventes aux enchères.

Les collections constituées comptent en général moins de 50 œuvres et le prix d'acquisition d'une œuvre le plus élevé reste inférieur à 5000 euros.

\section{En conclusion}

Les profils types proposés n'offrent qu'une photographie statique de l'investissement des collectionneurs dans la vie artistique au moment de l'enquête. Dans la réalité, ces investissements ne sont pas immuables et évoluent au fur et à mesure des « carrières » de collectionneurs. Au sein de chaque groupe que sont les quasi-professionnels, les investis, les pondérés ou les indépendants, se trouvent des individualités dont le profil peut se répartir le long d'un spectre défini autour des deux positions extrêmes suivantes.

D’un côté, des personnes pour lesquelles s'engager dans la vie artistique résulte $d^{\prime}$ une volonté première, celle de faire vivre et de valoriser leur collection. Dans ce cas, 
l'engagement se comprend davantage comme un moyen que comme une fin en soi, il a pour objet de faciliter la constitution de la collection. Par les informations que cet engagement fournit, par le réseau constitué, les collectionneurs améliorent leur capacité à trouver l'œuvre qui fait défaut à leur collection. Ces collectionneurs ont souvent un rapport décomplexé vis-à-vis du marché : ils achètent et vendent en utilisant les différents canaux disponibles (artistes, galeries, collectionneurs, ventes aux enchères). Les prêts effectués, la participation à la publication de catalogue assurent par ailleurs une visibilité aux artistes qu'ils détiennent. Ce faisant, par ces mêmes actions, ils contribuent à vitaliser la scène artistique.

De l'autre côté du spectre, des collectionneurs pour lesquels l'engagement résulte d'une volonté d'approcher au plus près le processus de création artistique. La possession que propose la collection résulte simplement de la volonté de soutenir des artistes émergents, et, dans cette configuration, c'est le rapport aux acteurs de la création artistique qui prime.

\section{Éléments de méthodologie}

En l'absence de statistiques nationales relatives à l'activité des collectionneurs, la population des collectionneurs d'art contemporain reste difficile à dénombrer, et donc à caractériser.

A été considérée comme collectionneur toute personne achetant régulièrement des œuvres, ce afin de ne pas introduire de biais dans la recherche en définissant a priori ce qu'est un «bon collectionneur* $»$.

Par « art contemporain » est entendue toute œuvre produite par un artiste vivant.

La difficulté de l'enquête tient au caractère privé de l'activité de collection : de ce fait, aucune source ne permet de connaître précisément l'étendue et le profil de la population mère des collectionneurs d'œuvres produites par des artistes vivants. Pour appréhender cette population et construire un échantillon suffisamment varié, notamment quant à la taille des collections, au temps dédié par les collectionneurs à leur passion, le questionnaire d'enquête a été largement diffusé auprès de différents réseaux : sociétés d'amis de musées parisiennes et en région, réseaux régionaux, institutionnels (Drac, Frac, écoles des beauxarts), galeries, relais individuels. Le questionnaire, strictement anonyme, était accessible en ligne et diffusé sous format PDF par courrier électronique (avec un engagement de respect de l'anonymat de la personne ayant rempli le formulaire) ou par courrier papier.

Le questionnaire tout comme la grille d'entretien étaient organisés autour de six thématiques: I'origine de la collection, l'information et les choix du collectionneur, les caractéristiques de la collection, la gestion de cette dernière, le collectionneur et le monde de l'art et enfin les profils individuels. Des entretiens qualitatifs semi-directifs ont ensuite été menés auprès de publics ciblés. L'enjeu était de toucher une pluralité de profils, audelà des collectionneurs les plus médiatisés.

L'enquête a été lancée en janvier et clôturée début juin 2014.

332 réponses ont été collectées et le profil type du collectionneur rejoint les caractéristiques dégagées par l'enquête internationale conduite en 2013 auprès d'une population de 900 collectionneurs d'art pour AXA (Collecting in the Digital Age, AXA Art Insurance, 2014).

\footnotetext{
* On peut distinguer dans la littérature académique deux approches de la définition du collectionneur. La première adopte un point de vue non utilitariste et quantitatif et considère que l'on devient collectionneur « quand on n'a plus de murs pour accrocher ses œuvres » (c'est-à-dire : « on devient collectionneur quand on ne considère pas l'œuvre comme objet décoratif »). La seconde procède d'une approche plus qualitative et accorde de l'importance au processus de sélection : « Le collectionneur procède d'un certain goût. »
} 
66 entretiens semi-directifs d'une durée moyenne d'une heure trente ont été conduits auprès de collectionneurs. Dix de ces collectionneurs ont été proposés par l'Association pour la diffusion internationale de l'art français (Adiaf), les autres ont été contactés grâce à des recommandations de galeristes ou de personnalités à la tête d'institutions nationales ou régionales tournées vers l'art contemporain. II pouvait également s'agir de personnes s'étant signalées en remplissant le questionnaire ou contactées grâce aux recherches effectuées individuellement par les chercheurs et grâce aux recommandations d'autres collectionneurs.

44 entretiens ont été conduits en région, 22 en île-de-France auprès de 47 hommes, 8 femmes et 11 couples (tableau A et B).

\section{Tableau A - Répartition par âge des personnes interrogées (entretiens semi-qualitatifs)}

\begin{tabular}{|lc|}
\hline Âge & Effectifs \\
30 à 40 ans & 8 \\
40 à 50 ans & 18 \\
50 à 60 ans & 18 \\
60 à 70 ans & 15 \\
Plus de 70 ans & 7 \\
\hline
\end{tabular}

Source: SACD/DEPS, Ministère de la Culture et de la Communication, 2014

\section{Tableau B - Répartition par profession des personnes interrogées (entretiens semi-qualitatifs)}

\begin{tabular}{|lc|}
\hline Profession & Effectifs \\
Commerçant & 6 \\
Chef d'entreprise & 14 \\
Cadre fonct. pub. et prof. intellectuelles & 6 \\
Cadre d'entreprise & 14 \\
Profession libérale & 17 \\
Profession intermédiaire & 4 \\
Autres & 5 \\
\hline
\end{tabular}

Source: SACD/DEPS, Ministère de la Culture et de la Communication, 2014 


\section{Abstract}

\section{Contemporary Art Collectors: Unsung Influential Figures on the Art Scene}

What is a collector? Although the French term collectionneur was first officially recorded in the dictionary in 1789', its definition covers a range of different practices: for some, the main emphasis is on an item's subjective value, whilst others focus more closely on the accumulation and selection process. Whether they collect shells, stamps, butterflies or works of art, collectors share a number of things in common. However, those who support "emerging" art have a more varied set of practices than those generally observed in their counterparts. In addition to simply being a buyer, the contemporary art collector has the ability to influence the artistic life, especially through the material support provided to artists and by contributing to the construction of artistic value. This particular characteristic is all the more notable as the collector's involvement in artistic life is not solely the prerogative of a narrow wealthy elite, as the collector profiles here demonstrate. Indeed it seems that collector practices may fall anywhere across a scale which ranges from the acquisitive collector, motivated by the desire to possess a work, to the actor collector, for whom the actual acquisition of a work is merely the expression of his willingness to contribute to the vitality of the artistic scene.

1. "Collector, -s (n). One who collects. Collection, -s (n). A number of objects gathered together under a common theme. A beautiful collection of paintings, books, antiques, medals, plants, shells, etc. » Dictionnaire de l'Académie française, 1789 (7th edition).

\section{À lire aussi}

\section{CE 2013-2}

\section{Les galeries d'art contemporain en France en 2012}

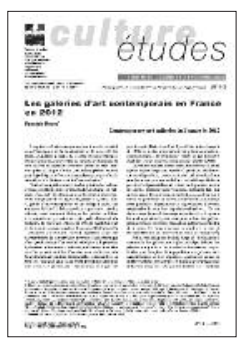

François Rouet

Janvier 2013, 12 p.

Les galeries d'art contemporain jouent un rôle essentiel dans l'émergence et la construction de la notoriété des artistes et, partant, dans la vitalité de la scène artistique française. Pourtant, l'analyse de leur activité est malaisée en l'absence d'une activité référencée dans la nomenclature d'activités française, ce qui oblige à croiser les sources. Létude a opté pour une méthodologie inédite qui permet de dénombrer et caractériser les galeries d'art contemporain. Inégalement réparties sur le territoire, regroupées à Paris (48 \% du total des galeries françaises), leur activité est significativement concentrée : $12 \%$ des galeries totalisent $72 \%$ du chiffre d'affaires annuel total. L'ancienneté de la galerie et sa présence sur le marché international sont des facteurs explicatifs importants de l'ampleur de son activité. Une typologie et une trajectoire type des galeries complètent l'analyse.

Directeur de la publication : Xavier Niel

Responsable de la publication : Edwige Millery

\section{Retrouvez l'ensemble des publications du DEPS sur : http://www.culturecommunication.gouv.fr/Etudes-et-statistiques et sur http://www.cairn.info}

Le DEPS n'assurant pas de diffusion physique de ses collections de synthèse, nous vous proposons de vous informer régulièrement des parutions par message électronique.

Pour ce faire, merci de bien vouloir nous communiquer votre courriel à l'adresse contact.deps@culture.gouv.fr 


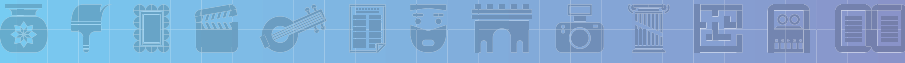

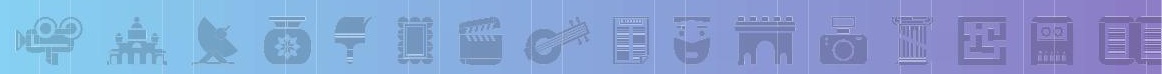

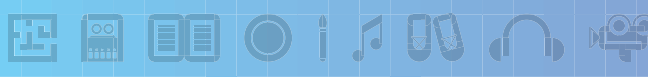

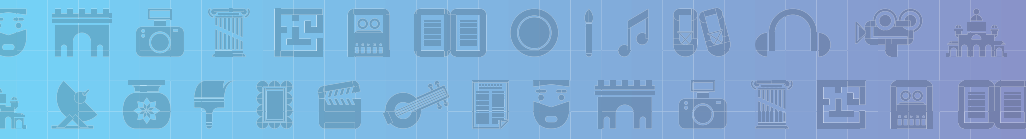

Qu'est-ce qu'un collectionneur? Si le nom a fait officiellement son entrée dans le dictionnaire en 1789 , sa définition recouvre des pratiques différentes : certaines approches mettent en avant la valeur subjective attachée à l'objet, tandis que d'autres soulignent l'importance du processus d'accumulation et de sélection. De nombreux traits semblent pouvoir définir communément les collectionneurs, quel que soit l'objet de la collection, coquillage, timbre, papillon ou encore ouvre d'art. Toutefois, les collectionneurs qui soutiennent l'art « en train de se faire » se distinguent par des pratiques plus variées que celles usuellement relevées chez leurs homologues. En effet, en plus d'être acquéreur, le collectionneur d'art contemporain est susceptible d'agir sur la vie artistique, notamment grâce au soutien matériel apporté aux artistes et en contribuant à la construction de la valeur artistique. Cette particularité est d'autant plus notable que l'engagement du collectionneur n'est pas l'apanage d'une seule élite fortunée, comme le montre la typologie des profils de collectionneurs établie ici.

Il apparaît en effet que les pratiques de collectionneurs se déploient le long d'un continuum allant du collectionneur acquéreur, mû par le besoin de posséder une œuvre, au collectionneur acteur, pour lequel l'acquisition n'est que l'expression de sa volonté de contribuer à la vitalité de la scène artistique. 